\title{
Optical/near-IR detection and monitoring of transients using SMARTS
}

\section{Dipankar Maitra*}

Univ. of Amsterdam

E-mail: dmaitra@science.uva.nl

\section{Charles Bailyn}

Yale University

E-mail: charles.bailyn@yale.edu

The SMARTS consortium uses four $1 \mathrm{~m}$ class telescopes at CTIO to carry out a number of wideranging astronomical projects, with a high priority towards monitoring long term time variability in stellar and non-stellar sources. We have an ongoing program routinely monitoring X-ray binaries simultaneously in optical and near-IR wavelengths. We discuss our observing strategies, instrumental capabilities/limitations, the possibilities of using SMARTS (by joining SMARTS or applying for observing via NOAO) for optical/near-IR detection as well as follow-up of new transients found by upcoming transient search projects.

Bursts, Pulses and Flickering:Wide-field monitoring of the dynamic radio sky June 12-15 2007 Kerastari, Tripolis, Greece

\footnotetext{
* Speaker.
} 


\section{Introduction}

The Small and Moderate Aperture Research Telescope System (SMARTS ${ }^{1}$ ) consortium operates four of the smaller telescopes at the Cerro Tololo Inter-American Observatory $\left(\mathrm{CTIO}^{2}\right)$. Current members of the consortium are American Museum of Natural History, University of Delaware, Fisk University, Georgia State University, National Optical Astronomy Observatory (NOAO), Ohio State University, Sejong University, Space Telescope Science Institute, State University of New York at Stony Brook, Vanderbilt University and Yale University. The observatory (CTIO) is located about $500 \mathrm{~km}$ north of Santiago (Chile), at an altitude of $\sim 2200$ metres on the Chilean Andes. With $\sim 88 \%$ useful nights and $\sim 75 \%$ photometric nights during November through March, and $\sim 70 \%$ useful and $\sim 50 \%$ photometric nights during April through October $^{3}$ and very little light pollution[2], the observatory still remains one of the best sites on earth. The telescopes operated by the SMARTS consortium are the $0.9 \mathrm{~m}, 1.0 \mathrm{~m}, 1.3 \mathrm{~m}$ and the $1.5 \mathrm{~m}$ ones. Fig. 1 shows a picture of the Tololo summit with the SMARTS telescopes labelled.

All four telescopes have different sets of instruments and detectors mounted on them, allowing a wide variety of projects that can be carried out, e.g.:

- Long-term monitoring of transients and other variable sources using the ANDICAM[1] instrument on the $1.3 \mathrm{~m}$.

- The user modes at the $0.9 \mathrm{~m}$ and $1.0 \mathrm{~m}$ allow doing precision astrometry and photometry using the standard filters as well as other narrow-band filters.

- The 20x20-arcminute FOV of the $4064 \times 4064$ Y4KCAM ${ }^{4}$ CCD on the $1.0 \mathrm{~m}$ allows wide-field imaging.

- The RC spectrograph on the $1.5 \mathrm{~m}$ telescope for low to moderate resolution $(300<R<3400$ in first order) spectroscopy.

The instruments and observing modes in which these telescopes currently operate are listed in Table 1. The rest of this article will be focussed mainly on the $1.3 \mathrm{~m}$ telescope because of its importance in long term monitoring capabilities.

\section{The 1.3m: Workhorse for Transient Monitoring}

The $1.3 \mathrm{~m}$ telescope was previously used as the southern telescope for the 2MASS project. Since 2003, the ANDICAM dual channel optical/near-IR imager has been permanently mounted on it. The choice of instrumentation as well as dedicated service mode observing makes the $1.3 \mathrm{~m}$ ideally suited for target-of-opportunity (ToO) observations as well as synoptic observations. Coordinated spectroscopy using the $1.5 \mathrm{~m}$ is also possible when appropriate.

\footnotetext{
${ }^{1} \mathrm{http} / / / \mathrm{www}$. astro.yale.edu/smarts

${ }^{2} \mathrm{http} / / /$ www.ctio.noao.edu

${ }^{3}$ Data taken from a compilation of historical record of sky conditions at CTIO, available at http://www.ctio.noao.edu/site/phot/sky_conditions.php and http://www.ctio.noao.edu/facil/s7/sec7.html

${ }^{4}$ http://www.astronomy.ohio-state.edu/Y4KCam/detector.html
} 


\begin{tabular}{lll}
\hline Telescope & Instrument & Observing Mode \\
\hline $0.9 \mathrm{~m}$ & 2K CCD & Alternating service and user \\
$1.0 \mathrm{~m}$ & 4K CCD & User only \\
$1.3 \mathrm{~m}$ & ANDICAM (OIR Imaging) & Monitoring queue only \\
$1.5 \mathrm{~m}$ & RCSpec (Spectroscopy) & Monitoring service/queue \\
\hline
\end{tabular}

Table 1: SMARTS telescopes, instruments and observing modes

\section{ANDICAM (A Novel Double-Imaging CAMera)}

With ANDICAM[1] one can obtain U, B, V, R, I photometry in optical (using a Fairchild 447 2048x2048 CCD with 15-micron pixels), and J, H, K photometry in near-IR (using a Rockwell $1024 \times 1024 \mathrm{HgCdTe}$ "Hawaii" array with 18-micron pixels). The pixel scale and the field of view of the optical CCD are 0!'369/pixel and $\sim 6^{\prime} \times 6^{\prime}$ respectively. The near-IR images are binned $2 \times 2$ to give a better pixel scale match to the seeing, giving a final pixel scale of $0.1274 /$ pixel and a field of view of $\sim 2$ '.4 $\times 2$ '.4 in J-, H- or K-band. A dichroic beam splitter inside the ANDICAM instrument allows simultaneous imaging at an optical band and a near-IR band.

\section{Scheduling and queue construction, ToO policy}

The sequential list of targets to be observed every night at the $1.3 \mathrm{~m}$ is created every day at Yale by the queue manager, and sent to the observers by late afternoon. This list, called the queue, follows merged institutional priority lists of targets. ToOs are triggered if they have higher priority than scheduled program or if scheduled program is not seriously inconvenienced. Unpredictable events requiring rapid response (e.g. GRB), and similar events which cause interruptions after the queue is received by the observer at the telescope can only be observed by prior arrangement (i.e. notifying in the proposal about the possibility of an interruption).

\section{Data pipeline and calibration}

Data is taken by service observers at the telescope. This comprises not only observing the science targets, but also a standard set of calibration frames. Since taking calibration flat frames for all the UBVRIJHK bands is not feasible every day, a smaller subset of flats are obtained every day and the subset changed from day to day so as to cover all the filters at least once every three days. These calibration frames are given to every observer at no cost. Additionally, on each photometric night, one Landolt field is observed once in all optical filters and two near-IR standards are observed once in all IR filters. An attempt is made to observe each standard field at its best airmass. Once a month, more thorough observations of the same standards are performed, observing each of the 3 standard fields (1 Landolt, 2 IR fields) at 3 different airmasses. From these observations photometric zeropoints and coefficients in BVRI are calculated and posted on the web for all observers $^{5}$. The observations of these standard stars are also freely available to the users.

\footnotetext{
${ }^{5}$ http://www.astro.yale.edu/smarts/smarts $13 \mathrm{~m} /$ photometry.html
} 
Once science targets are observed, first glance comparisons and quick aperture photometry is done for the known sources by the observers on-site. Nightly data is SFTP-d to the SMARTS central server at Yale (New Haven) the next morning for pipelined processing and archiving. The optical data is fully calibrated and the near-IR binned to $2 \times 2$, and made available to the observers prior to noon (Eastern time). In near future NOAO hopes to set up a pipeline/archive on mirrored sites in La Serena (Chile) and Tucson (USA).

\section{Science programs at the $1.3 \mathrm{~m}$}

A brief list of the ongoing science programs at the $1.3 \mathrm{~m}$ is given below:

- X-ray binaries and optical/near-IR follow up of GRBs (Yale/Amsterdam; see e.g Fig. 目 and Fig. 3)

- Microlensing events and lensed quasar time delays (OSU)

- Nearby supernovae (NOAO community)

- Solar system objects (Yale/NOAO/Louisiana State University)

- QSO monitoring (GSU/Chile)

- $\mathrm{CV}$ monitoring (Chile/NOAO)

- Novae (SUNY/STScI)

- Pre-main sequence star variability (Vanderbilt/SUNY)

\section{FAQ}

- What is the possibility of joining SMARTS?

You are welcome to join SMARTS. Consortium membership changes annually as scientific and financial considerations evolve.

- Do I have to be a SMARTS member to use these telescopes?

No, you can still apply through NOAO. Currently NOAO has $25 \%$ time on the SMARTS telescopes. Anyone can submit an NOAO proposal ${ }^{6}$ to request observations using the SMARTS telescopes. Oversubscription via NOAO is fairly low $(<1.5)$.

- How much does it cost to run SMARTS?

About $\$ 1000$ per user night and $\$ 1500$ per service night.

\section{Acknowledgments}

DM would like to thank Michelle Buxton for providing the light curves of GX 339-4 ahead of publication. DM also thanks the Leids Kerkhoven-Bosscha Fonds (LKBF) grant 07-052 of the Netherlands. It is a pleasure to acknowledge the warm hospitality of the LOC as well as the people of the village of Kerastari.

\footnotetext{
${ }^{6} \mathrm{http} / / /$ www.noao.edu/gateway/propinfo.html
} 


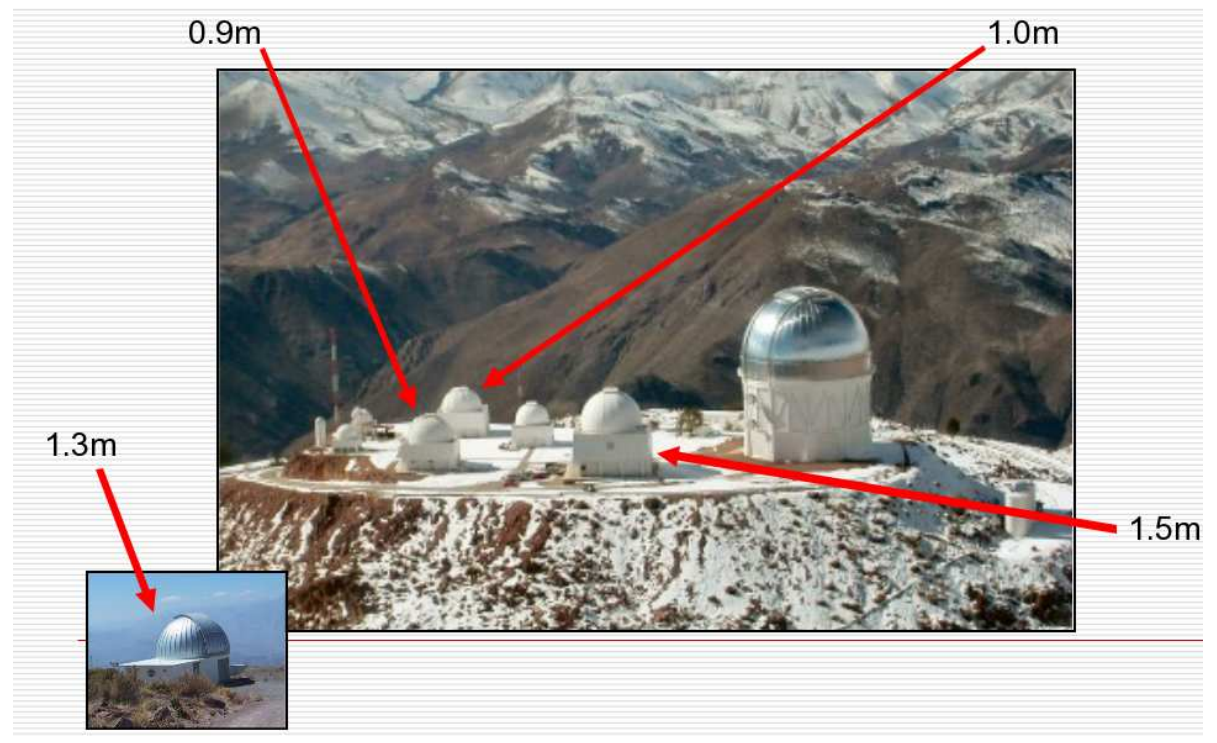

Figure 1: Telescopes on the Tololo summit. The Blanco $4 \mathrm{~m}$ dominates the scene. The telescopes operated by the SMARTS consortium are labelled. The 1.3m (ex-2MASS southern telescope) is actually not on the summit itself, but few tens of metres below, and shown separately. Photographs courtsey of NOAO/AURA/NSF and Fred Walter/SMARTS.

\section{References}

[1] DePoy, D. L., et al. 2003, Proceedings of the SPIE, 4841, 827

[2] Krisciunas, K., Semler, D. R., Richards, J., Schwarz, H. E., Suntzeff, N. B., Vera, S., \& Sanhueza, P. 2007, PASP, 119, 687 


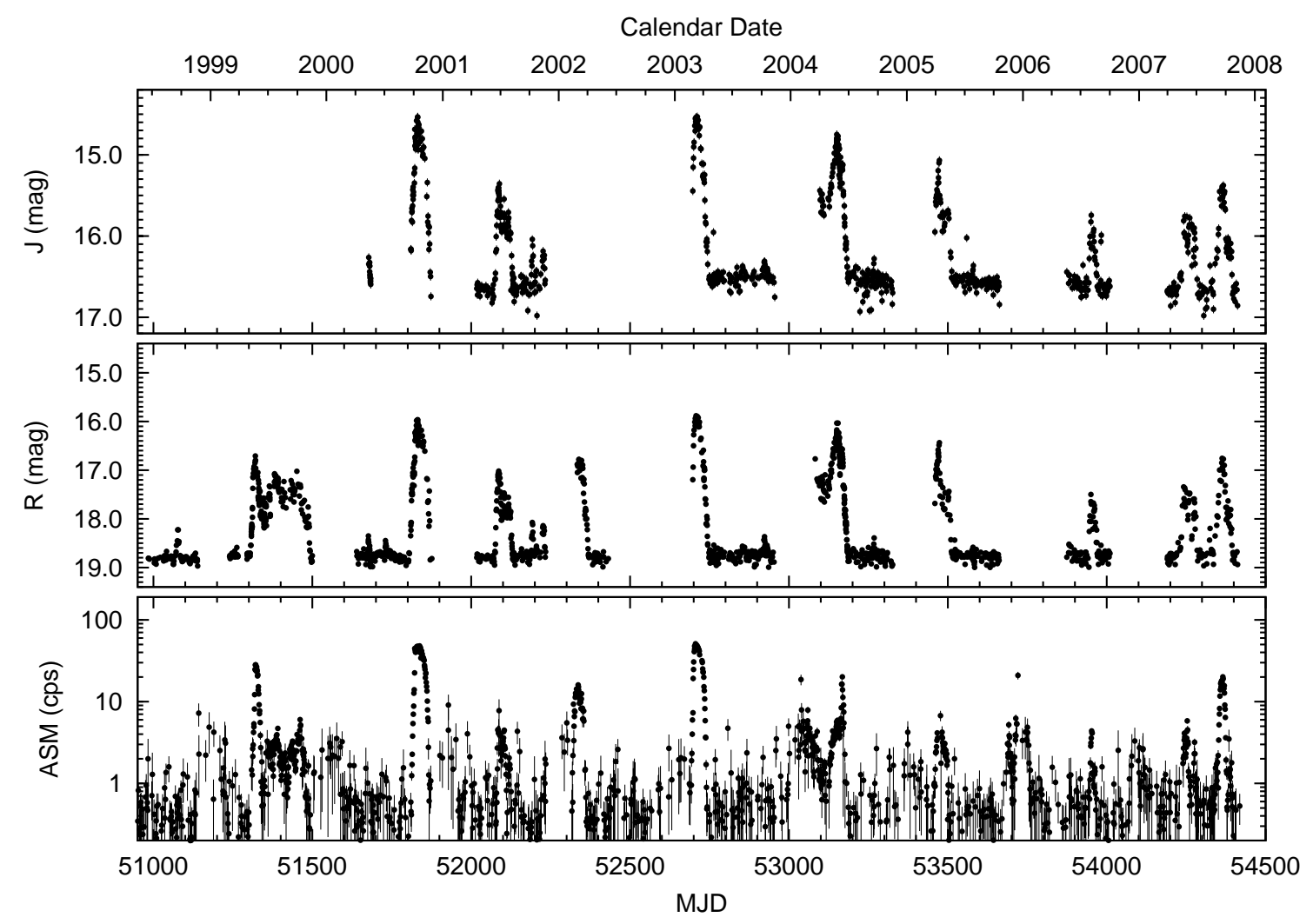

Figure 2: Multi-wavelength long-term monitoring of the neutron star soft X-ray transient binary system Aql X-1 since 1998. Top panel: J-band data from the $1.3 \mathrm{~m}$ since the ANDICAM was installed at the $1.3 \mathrm{~m}$ in 2003. Middle panel: R-band data from $1.0 \mathrm{~m}$ and $1.3 \mathrm{~m}$ since June 1998. Bottom panel: Soft X-ray flux between $1.5-12 \mathrm{keV}$ measured by RXTE/ASM satellite in ASM count-rate (1 Crab = 75.5 ASM cps) 


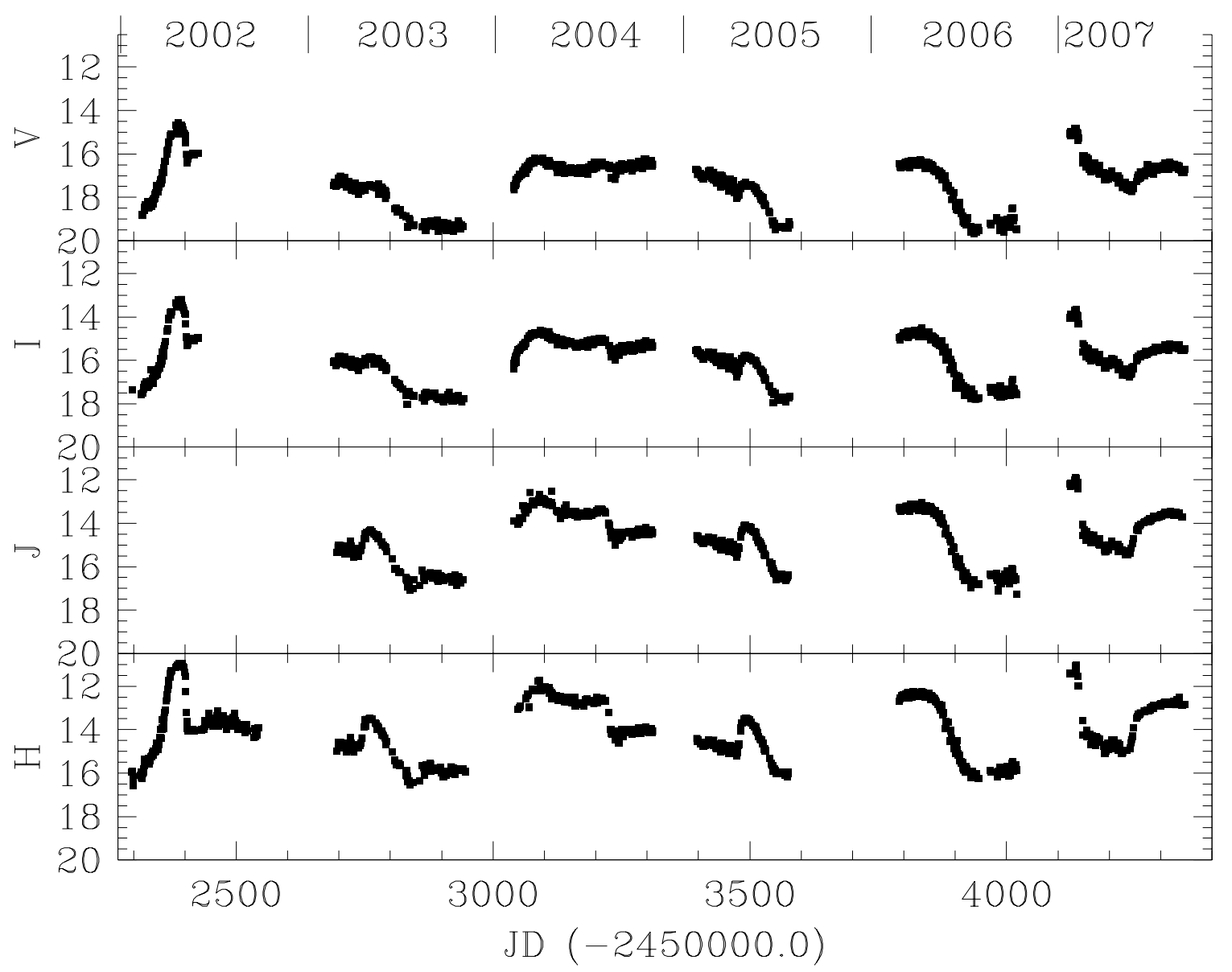

Figure 3: Long-term monitoring of the black hole candidate GX 339-4 using SMARTS since 2002. V, I, $\mathrm{J}$ and H-band light curves are shown in the panels from top to bottom. GX 339-4 light curves courtsey Michelle Buxton. 